\title{
Protein Kinase Protein Phosphorylation
}

National Cancer Institute

\section{Source}

National Cancer Institute. Protein Kinase Protein Phosphorylation. NCI Thesaurus. Code C18970.

Protein Kinase Protein Phosphorylation involves covalent linkage of a phosphate group (from a donor compound such as ATP) with a serine, threonine, or tyrosine residue of a Protein Kinase acceptor, often as a mechanism of kinase activity regulation. 